\title{
Palm Vein Recognition with Local Binary Patterns and Local Derivative Patterns
}

\author{
Leila Mirmohamadsadeghi and Andrzej Drygajlo \\ Swiss Federal Institude of Technology Lausanne (EPFL) \\ CH-1015 Lausanne, Switzerland \\ \{leila.mirmohamadsadeghi, andrzej.drygajlo\}@epfl.ch
}

\begin{abstract}
Palm vein feature extraction from near infrared images is a challenging problem in hand pattern recognition. In this paper, a promising new approach based on local texture patterns is proposed. First, operators and histograms of multi-scale Local Binary Patterns (LBPs) are investigated in order to identify new efficient descriptors for palm vein patterns. Novel higher-order local pattern descriptors based on Local Derivative Pattern (LDP) histograms are then investigated for palm vein description. Both feature extraction methods are compared and evaluated in the framework of verification and identification tasks. Extensive experiments on CASIA Multi-Spectral Palmprint Image Database V1.0 (CASIA database) identify the LBP and LDP descriptors which are better adapted to palm vein texture. Tests on the CASIA datasets also show that the best adapted LDP descriptors consistently outperform their LBP counterparts in both palm vein verification and identification.
\end{abstract}

\section{Introduction}

Intrinsic physiological patterns are naturally hard to observe and therefore offer a high degree of privacy and security for biometric recognition. In this context, the palm veins have emerged as a promising new biometric modality [16]. The palm vein imaging requires near-infra-red (NIR) illumination for extracting the complex vascular structures residing inside the palm.

A key issue in palm vein feature extraction from NIR images is finding efficient descriptors for palm vein appearance. Different methods such as Hessian phase, localized Radon transform, ordinal code, Laplacianpalm based on Principal Component Analysis, complex matched filtering, repeated line tracking have been studied for palm vein, dorsal hand vein and finger vein recognition [18, 4]. Lately, local texture descriptors have also gained attention in finger vein and dorsal hand vein recognition.

A good texture descriptor is one of the key issues for a well designed palm vein feature extraction method. Find- ing good descriptors for the appearance of local palm vein regions is an open issue. To the best of our knowledge, no local texture pattern operators have been investigated for palm vein representation.

The texture analysis researchers have developed a variety of different descriptors for the appearance of image patches, e.g., Local Binary Patterns (LBPs) and their variants such as multi-scale LBPs, multi-scale block LBPs, rotation-invariant LBPs, etc., and Local Derivative Patterns (LDPs) and their higher order variants. The LBP operator has been proposed for face recognition [2, 3], finger vein recognition [8], dorsal hand vein recognition [14, 15] and palm-print recognition [10]. The LDP operator has been proposed for face recognition [17] and finger vein recognition $[7,6]$. However, palm vein recognition problem has not been investigated from such point of view.

This paper investigates two new feature extraction approaches based on a variety of multi-scale Local Binary Patterns (LBPs) and high-order Local Derivative Patterns (LDPs), in order to identify the best descriptors for palm veins.

The rest of the paper is organized as follows. In Section 2, we introduce and discuss operators and histograms of multi-scale LBPs to identify new efficient descriptors for palm vein patterns. Novel high order local pattern descriptors based on LDP histograms are then investigated for palm vein representation in Section 3. In Section 4, the dataset used for experiments, as well as the extraction of the region of interest and pre-processing operations are established. In Section 5 both feature extraction methods are compared and evaluated in the framework of verification and identification tasks. Finally, conclusions are drawn in Section 6 with some discussions.

\section{Local Binary Patterns}

\subsection{Original LBP}

The Local binary pattern (LBP) operator is a texture descriptor originally proposed in 1994 [11]. This operator is based on the gray level comparison of a neighborhood of 
pixels. The original operator considers a $3 \times 3$ neighborhood of 8 pixels around a center pixel. This neighborhood is thresholded by the value of the center pixel and the result is considered as a binary number or its decimal equivalent (Equation 1).

$$
L B P_{P, R}\left(I_{c}\right)=\sum_{p=1}^{P} s\left(Z_{p}-Z_{c}\right) 2^{p-1},
$$

where $s(u)=1$ if $u \geq 0$ and 0 otherwise. Each resulting decimal number is considered as a type of micro-pattern. These micro-patterns are often represented in histograms whose bins each contain one type of pattern. The LBP operator was later modified to be sensitive to certain types of spatial patterns. In particular the "uniform" LBP places emphasis on patterns having at most two bitwise transitions. Other modifications include rotation-invariance and graylevel invariance [12]. The size of the operator is defined as the number of neighboring pixels $P$ at radius from center point $R$ (Figure 1).
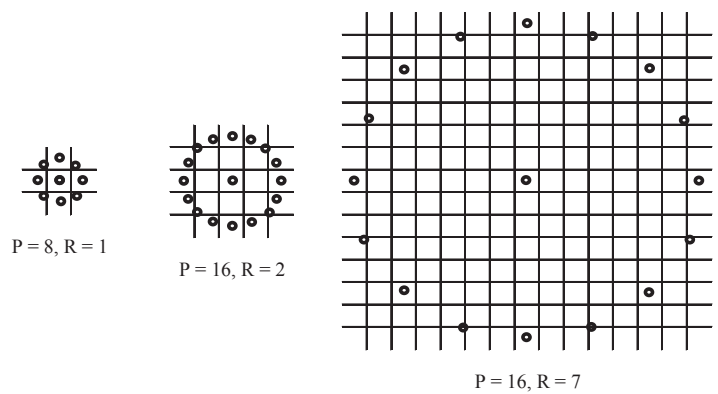

Figure 1. LBP neighborhood sizes.

\subsection{Palm Vein Description with LBP}

Palm veins are line structures with changing width, whose gray-level values differ from the background. The LBP operator is based on gray-level differences in local neighborhoods. Therefore it has the potential to extract discriminative features from palm vein images. The size of the operator must be adapted to the size of the information to be extracted. In the case of a neighborhood containing a vein region, the vein will either cross the local neighborhood or end inside. Thus, the resulting patterns of interest will not present many discriminative bitwise transitions indicating gray-level changes. It is therefore logical to consider "uniform" patterns. The direction of veins presents a discriminative feature, therefore it is not necessary to consider rotation-invariant patterns. In order to preserve local spatial information, the LBP operator is applied on partitions of an image and not to the whole image (Figure 3 ). The histograms resulting from individual portions are concatenated to create a descriptor over the whole image. The size of neighborhoods $(P, R)$ and the number of sub-images are the prime parameters to be determined to best extract discriminative vein information. In this study, the most efficient operator for palm vein images of the size $236 \times 236$, with vein width of about 2-10 pixels is the uniform LBP with parameters $P=16, R=7$ applied on 16 sub-images of size $59 \times 59$. A smaller number of neighboring points is beneficial in reducing noise feature extraction. Where the neighbors specified by $P$ and $R$ do not correspond to a single image pixel, the concerned pixels are averaged with weights depending on their position with respect to the circle of radius $R$. This LBP operator is applied on overlapping $15 \times 15$ blocks (Figure 2 ) whose center pixels are subimage pixels from rows 8-52 and columns 8-52 (to avoid border effects). Given the neighbors considered in Figure 2

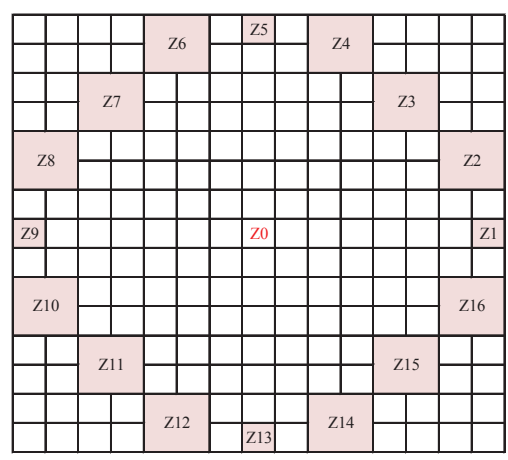

Figure 2. (16,7) LBP neighborhood.

around a center pixel $Z_{0}$, the LBP code for one neighborhood is obtained by Equation 2.

$$
\text { code }=\sum_{i=1}^{16} s\left(Z_{i}-Z_{0}\right) 2^{i-1}
$$

The obtained code is saved by incrementing the corresponding histogram bin. The code corresponding to the application of LBP on a sub-image results in a histogram with 240 bins (uniform LBP length $=P(P-1$ ). The corresponding mapping table is used to asign bins to decimal codes). The 16 histograms (each for one sub-image) are concatenated to create a descriptor of length 3840 over a whole image (Figure 3).

\section{Local Derivative Patterns}

\subsection{Original LDP}

The Local derivative pattern (LDP) operator [17] is a high-order texture descriptor. It was proposed as an encoding scheme for local patterns, initially for face recognition in comparison with the LBP method, and was shown to be more efficient. The LBP operator extracts first order 


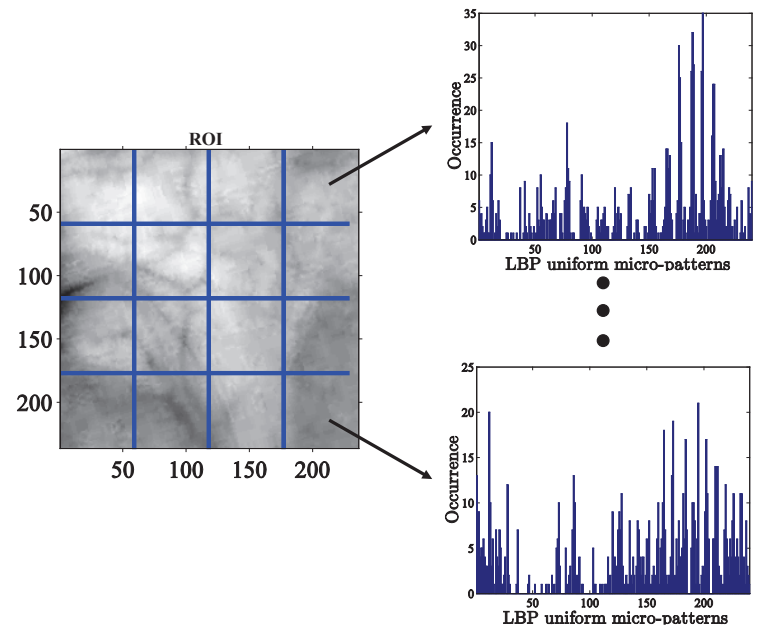

Figure 3. LBP histograms over sub-images.

non-directional patterns. On the other hand the LDP operator extracts the derivative direction variation information which is regarded as second order pattern information. Each neighboring pixel contributes to the pattern code with the direction of its derivative with respect to the derivative of the center point. The derivatives are considered in different directions, in the original case, the directions 0, 45, 90 and 135 degrees were considered. The image derivatives in each direction are obtained by subtracting neighboring pixels according to the direction (Equation 3 ) for the case of the first image derivatives). In an $n$-order LDP, the $(n-1)$ th order derivative is considered.

$$
\begin{aligned}
& I_{0^{\circ}}^{\prime}\left(Z_{0}\right)=I\left(Z_{0}\right)-I\left(Z_{4}\right) \\
& I_{45^{\circ}}^{\prime}\left(Z_{0}\right)=I\left(Z_{0}\right)-I\left(Z_{3}\right) \\
& I_{90^{\circ}}^{\prime}\left(Z_{0}\right)=I\left(Z_{0}\right)-I\left(Z_{2}\right) \\
& I_{135^{\circ}}^{\prime}\left(Z_{0}\right)=I\left(Z_{0}\right)-I\left(Z_{1}\right)
\end{aligned}
$$

The neighbors $Z_{1}, \ldots, Z_{8}$ are considered according to Figure 4 around a center pixel $Z_{0}$. The direction compliance with

$$
\begin{array}{|l|l|l|}
\hline \mathrm{Z} 1 & \mathrm{Z} 2 & \mathrm{Z3} \\
\hline \mathrm{Z} 8 & \mathrm{Z} 0 & \mathrm{Z} 4 \\
\hline \mathrm{Z7} & \mathrm{Z} 6 & \mathrm{Z} 5 \\
\hline
\end{array}
$$

Figure 4. 8-neighborhood considered in LDP.

the derivative value at the center point is encoded for every neighbor (Equation 6). This operation is repeated for each considered derivative direction as follows:

$$
f\left(I_{\alpha}^{\prime}\left(Z_{0}\right), I_{\alpha}^{\prime}\left(Z_{i}\right)\right)= \begin{cases}0 & \text { if } I_{\alpha}^{\prime}\left(Z_{0}\right) \cdot I_{\alpha}^{\prime}\left(Z_{i}\right)>0 \\ 1 & \text { if } I_{\alpha}^{\prime}\left(Z_{0}\right) \cdot I_{\alpha}^{\prime}\left(Z_{i}\right) \leq 0\end{cases}
$$

where $i=1,2, \ldots, 8$ are the indices of the neighbors. The LDP pattern code for a given direction is the concatenation of the bits corresponding to each neighbor (Equation 5).

$$
\begin{gathered}
\operatorname{LDP}_{\alpha}^{2}\left(Z_{0}\right)=\left\{f\left(I_{\alpha}^{\prime}\left(Z_{0}\right), I_{\alpha}^{\prime}\left(Z_{1}\right)\right), \ldots,\right. \\
\left.f\left(I_{\alpha}^{\prime}\left(Z_{0}\right), I_{\alpha}^{\prime}\left(Z_{8}\right)\right)\right\}
\end{gathered}
$$

As with the case of the LBP descriptors, the patterns extracted with the LDP operator are stored in histograms with their decimal values (Equation 6) over the image or portions of the image. Each bin of a histogram contains the occurrence of a given micro-pattern.

\subsection{Palm Vein Description with LDP}

As in the case of fitting the LBP operator to best extract veins, the LDP operator needs to be applied with parameters best suitable for the vein extraction task. The order of the operator $n$, the derivative directions, the scale (radius from center point) applied to the neighborhood size and the number and size of sub-image blocks on which the LDP operator is directly applied, are varied. In this study, the best operator on images of size $236 \times 236$ with veins of $2-10$ pixels thick is the third order LDP at a radius of 6 pixels from the center point (Figure 5). It is applied in directions 0, 45, 90 and 135 degrees on 16 sub-images.

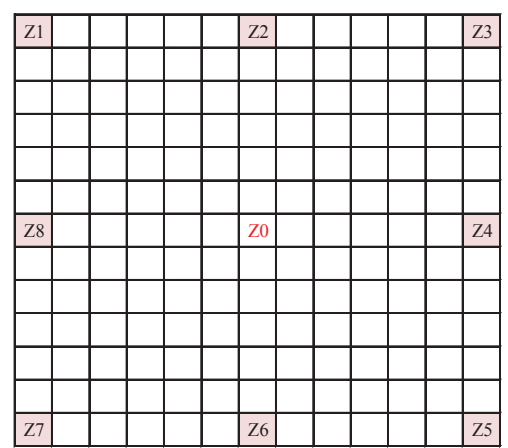

Figure 5. Neighborhood considered in third-order LDP at scale 6.

Given the neighbors considered in Figure 5, the LDP code for one neighborhood for a given scale is obtained by Equation 6 , where $Z_{0}$ and $Z_{i}, i=1, \ldots, 8$ are the considered neighbors.

$$
\text { code }=\sum_{i=1}^{8} f\left(I_{\alpha}^{\prime}\left(Z_{0}\right), I_{\alpha}^{\prime}\left(Z_{i}\right)\right) 2^{8-i}
$$

The extracted codes for all overlapping neighborhoods in a sub-image are binned in a histogram and the histograms concatenated over the image to produce an image descriptor (Figure 6). The number of histogram bins is computed as number of scales $\cdot$ number of directions $\cdot 2^{8}$ which yields 1024 bins for four directions. For an image divided into 16 sub-images, the image descriptor is of length 16384. 


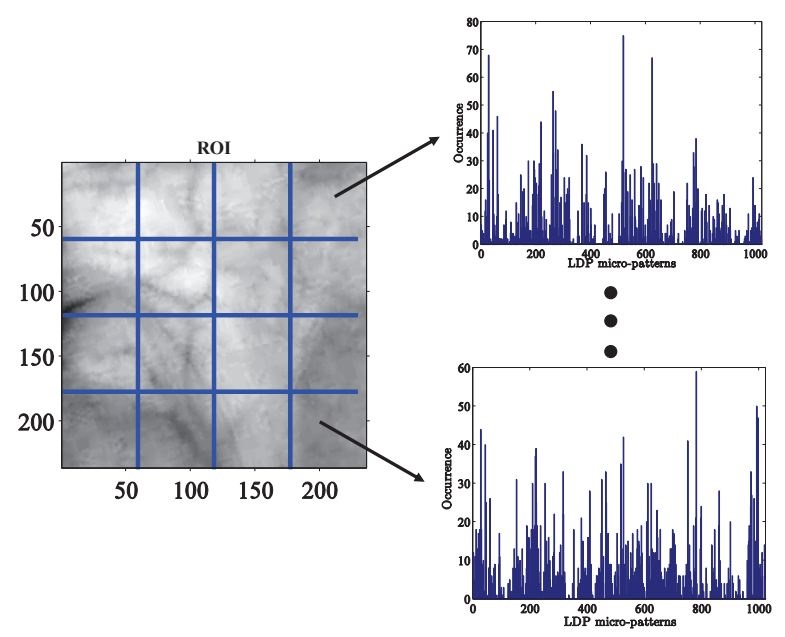

Figure 6. LDP histogram over image blocks.

\section{Data}

\subsection{Dataset}

The dataset used in this work for training and testing the algorithms is the CASIA multi-spectral Palmprint Image Database V1.0 (CASIA database) [1]. This dataset consists of palm print images of 100 individuals (six samples per individual), captured under six different NIR illuminators. Palm veins are most visible under the illuminator at 940 $\mathrm{nm}$ wavelength. The sub-set used here contains all samples from all individuals left hands under $940 \mathrm{~nm}$ illuminator.

\subsection{Region of Interest}

A region of interest (ROI) is extracted from every NIR palm image, on which pre-processing and feature extraction will be performed. Selecting a rectangular shape region is most practical given the LBP and LDP neighborhood requirements, and contains the most interesting region of the palm veins. In this study, the ROI is defined geometrically with respect to the four fingers. The hand contour is first found, then convex points complying geometrically to the position of in-between-finger points are kept as reference points. These reference points and the segments linking them are used to define a square ROI (Figure 7). In such a ROI, two different images of the veins of a same person are aligned. For the LBP and LDP operators to be efficient, it is important the veins are aligned in two different images of a same person.

\subsection{Pre-processing}

The ROI is defined with respect to reference points whose position is unique for every hand. Therefore its size will vary for different individuals and even different captures of the same individual if the hand is placed at different

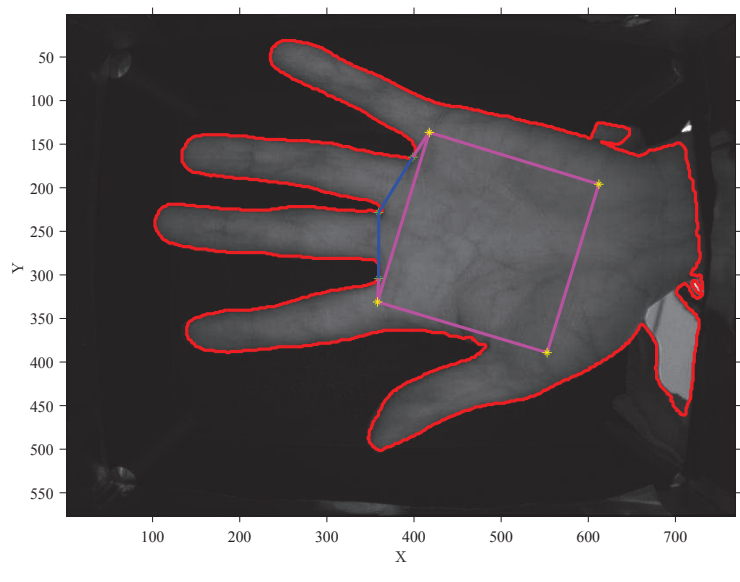

Figure 7. ROI extraction.

distances from the sensor during the acquisitions. In order to cope with the size difference, all ROIs are re-scaled with bicubic interpolation to a fixed size (the average size of ROIs extracted from the data-set). Descriptors are extracted from the re-scaled version of the ROI $(236 \times 236)$. This re-scaling ensures that veins are aligned in images of a same person at different distances from the sensor. The re-scaling ratio is a quality indicator, since images whose scales are very different have a greater chance of inducing an error in the comparison process. The LBP and LDP operators are based on gray-level difference of neighboring pixels, therefore they do not require further pre-processing and enhancement of vein patterns. For LBP however, simple pre-processing with adaptive histogram equalization shows systematic improvement in the performance of this operator.

\section{Experiments and Results}

In palm vein recognition, the extracted descriptor of a person is compared to one or several descriptors. In verification, False Non-Match Rate (FNMR) and False Match Rate (FMR) are evaluation metrics which are represented in Receiver Operating Characteristic (ROC) curves. In identification, the Correct Identification Rate (CIR) is the evaluation metric. It describes overall performance in the Cumulative Match Characteristic (CMC) curve [9]. In this study, the verification and closed-set identification tasks are considered. Leave-one-out technique is used in verification and the nearest neighbor search method is used in identification. Different measures are used to provide a similarity measure for two descriptors, such as $\chi^{2}$ measure, Bhattacharyya coefficient [5] and histogram intersection [13]. It was found that histogram intersection (Equation 7) performs slightly better than other metrics in the palm vein recognition task, therefore only the results obtained with this measure are 
presented in this section.

$$
H(p, q)=\frac{\sum_{i} \min \left(p_{i}, q_{i}\right)}{\sum_{i} q_{i}}
$$

In Equation 7, $p$ and $q$ are the two histograms being compared, of $i$ bins each.

\subsection{LBP Operator Choices}

The LBP operators ${ }^{1}$ are tested with different sizes, the highest CIR obtained for the images of the dataset is the $L B P_{(16,7)}$ (Table 1). Different sub-image sizes were considered, 16 square sub-images results in the best performance (Table 2).

\begin{tabular}{|c|c|c|c|c|c|c|c|}
\hline $\mathrm{P} \quad \mathrm{R}$ & 1 & 4 & 5 & 6 & 7 & 8 & 9 \\
\hline 8 & $70 \%$ & $87.4 \%$ & $88.8 \%$ & $88.6 \%$ & $89.4 \%$ & $89.8 \%$ & $90.6 \%$ \\
\hline 16 & $69.4 \%$ & $88.4 \%$ & $91.2 \%$ & $91.2 \%$ & $93 \%$ & $92.8 \%$ & $92 \%$ \\
\hline
\end{tabular}

Table 1. CIR for LBP operators with uniform mapping tested for different neighborhood sizes in the closed-set identification task.

\begin{tabular}{|l|l|l|l|}
\hline row partitions col partitions & 2 & 4 & 8 \\
\hline 2 & $80.4 \%$ & $85.2 \%$ & $79 \%$ \\
\hline 4 & $88.4 \%$ & $\mathbf{9 1 \%}$ & $84.2 \%$ \\
\hline 8 & $81.8 \%$ & $84.6 \%$ & $80.2 \%$ \\
\hline
\end{tabular}

Table 2. CIR for LBP operators with uniform mapping tested for different sub-image sizes in the closed-set identification task.

Custom mapping: To compress the uniform mapping, a custom mapping learned from the training dataset can be applied in order to reduce the number of descriptor elements. A mapping table was created based on the mean and standard deviation of bins, over descriptors from 10 persons. A CIR of $86 \%$ is obtained with a descriptor of length 42 extracted by the $\operatorname{LBP}_{(16,7)}$ over a sub-image (the image is divided into 16 square sub-images).

\subsection{LDP Operator Choices}

The LDP operator ${ }^{2}$ is tested for different orders, scales and directions, the highest CIR obtained for the images of the dataset is the third order LDP at scale 6 in directions 0 , 45, 90 and 135 degrees (Tables 3, 4 and 5). Different subimages sizes were considered, 16 square sub-images results in the best performance (Table 6).

\begin{tabular}{|l|l|l|l|}
\hline Order & 2 & 3 & 4 \\
\hline CIR & $94.8 \%$ & $\mathbf{9 7 \%}$ & $93.2 \%$ \\
\hline
\end{tabular}

Table 3. CIR for LDP operators tested for different orders at scale 6 in directions $0,45,90$ and 135 degrees in the closed-set identification task.

\footnotetext{
${ }^{1}$ MATLAB code in part by M. Heikkila and T. Ahonen.

${ }^{2}$ MATLAB code in part by $\mathrm{S}$. Paris.
}

\begin{tabular}{|l|l|l|l|l|}
\hline Scales & 5 & 6 & 7 & 8 \\
\hline CIR & $95.4 \%$ & $\mathbf{9 7 \%}$ & $96.6 \%$ & $95 \%$ \\
\hline
\end{tabular}

Table 4. CIR for third-order LDP operators tested for different scales in the directions $0,45,90$ and 135 degrees in the closedset identification task.

\begin{tabular}{|l|l|l|l|l|}
\hline Directions & 0 & 0,45 & $\begin{array}{l}0, \\
45, \\
90\end{array}$ & $\begin{array}{l}0, \\
45, \\
90, \\
\end{array}$ \\
& & & & 135 \\
\hline CIR & $88.8 \%$ & $95 \%$ & $96.2 \%$ & $\mathbf{9 7 \%}$ \\
\hline Directions & 0, & 180, & 0, & 45, \\
& $45, \ldots$, & 225, & 90, & 135, \\
& 315 & 270, & 180, & 225, \\
& & 315 & 270 & 315 \\
\hline CIR & $96.8 \%$ & $96.6 \%$ & $95.8 \%$ & $95.6 \%$ \\
\hline
\end{tabular}

Table 5. CIR for third-order LDP operators tested for combinations of different orientations at scale 6 in the closed-set identification task.

It is observed that the third-order LDP operator performs best when applied at a scale 6 . With respect to the derivative direction choice, the best performance is obtained when one representative of each direction axis is present ( 0 or 180,45 or 225,90 or 270,135 or 315 degrees), in particular, the option $0,45,90,135$ degrees performs most successfully.

\begin{tabular}{|l|l|l|l|}
\hline row partitions col partitions & 2 & 4 & 8 \\
\hline 2 & $92.6 \%$ & $95 \%$ & $87 \%$ \\
\hline 4 & $96.6 \%$ & $\mathbf{9 7 \%}$ & $88.6 \%$ \\
\hline 8 & $87.8 \%$ & $89.6 \%$ & $76.8 \%$ \\
\hline
\end{tabular}

Table 6. CIR for third-order LDP operators tested for different subimage sizes at scale 6 in directions 0, 45, 90 and 135 degrees in the closed-set identification task.

Clearly, 16 sub-images yields in the best performance compared to other partitioning schemes.

\subsection{Verification}

In the verification task, the histogram extracted from the query image is compared to the stored histogram of the claimed identity. The overall verification system is assessed with the ROC curve, generated by varying the threshold on the histogram intersection measure (Figure 8). The Equal Error Rate (EER) point (where FNMR = FMR) is 0.004 for the LBP operator and 0.0009 for the LDP operator.

\subsection{Identification}

In the identification task, the histogram extracted from the query image is compared to all the stored histograms to determine which one it is most similar to. A nearest neighbor search with the histogram intersection distance metric is applied to determine identity among enrolled individuals. The overall identification system performance is assessed with the CMC curve over the dataset (Figure 9). 


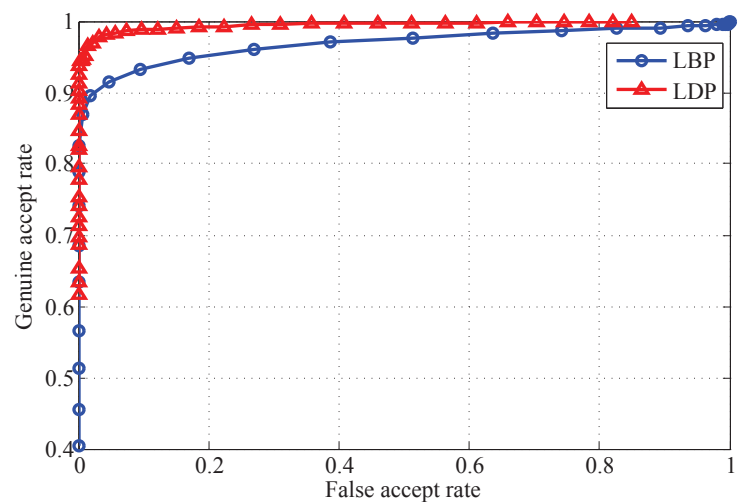

Figure 8. ROC curve for the verification task over the database of left hands. $L B P_{(16,7)}$ and third-order LDP at scale 6 in directions $0,45,90,135$ degrees are used.

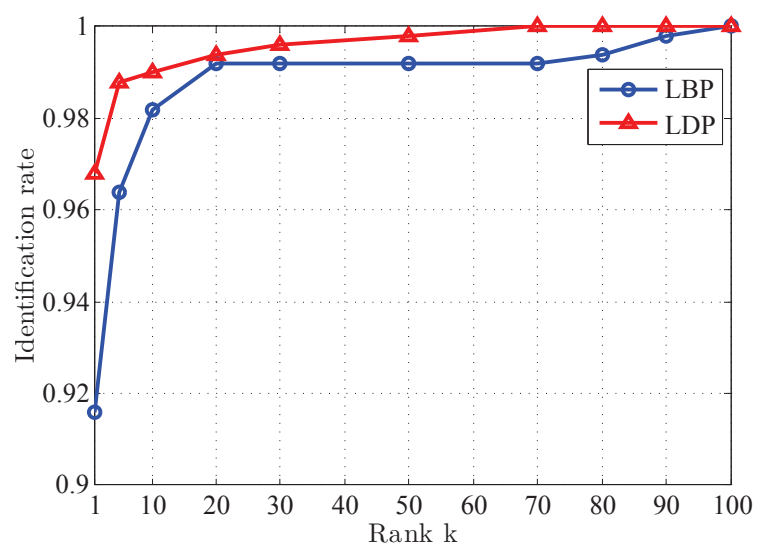

Figure 9. CMC curves for the identification task using $L B P_{16,7}^{u 2}$ and third order LDP in scale 6 and directions $0,45,90$ and 135 degrees.

\subsection{Throughput}

The MATLAB implementations used in this study extracts a descriptor in 10-40 ms on a machine with a Intel(R) Core(TM) i7 CPU @ 2.67 GHz with 4.00 GB RAM.

\section{Conclusions and Future Work}

In this study, palm vein recognition with two local texture description methods was performed. The two feature extraction operators Local Binary Patterns (LBPs) and Local Derivative Patterns (LDPs) were investigated in terms of their ability to adapt to palm vein description. They were adjusted based on discriminative features identified in vein texture to be used as image descriptors in the context of recognition with palm veins. These operators present computational simplicity and efficiency and proved to be well adapted to description for palm vein recognition. The LDP operator was found to perform better than the LBP operator in verification and identification tasks.

The use of a custom mapping table to reduce the size of image descriptors also proved promising. Future work will include investigating techniques to choose the bins representing the most discriminative information in the palm vein image set and create a custom mapping to reduce the size of image descriptors.

\section{References}

[1] http://www.cbsr.ia.ac.cn/MS_Palmprint Database.asp. 4

[2] T. Ahonen, A. Hadid, and M. Pietikäinen. Face recognition with local binary patterns. In Computer Vision - ECCV 2004, LNCS 3021, pages 469-481. Springer Berlin / Heidelberg, 2004. 1

[3] T. Ahonen, A. Hadid, and M. Pietikäinen. Face description with local binary patterns: Application to face recognition. IEEE Transactions on Pattern Analysis and Machine Intelligence, 28:2037-2041, 2006. 1

[4] M. Greitans, M. Pudzs, and R. Fuksis. Palm vein biometrics based on infrared imaging and complex matched filtering. In Proceedings of the 12th ACM Workshop on Multimedia and Security, pages 101-106, ACM, New York, 2010. 1

[5] T. Kailath. The Divergence and Bhattacharyya Distance Measures in Signal Selection. IEEE Transactions on Communication Technology, 15(1):52 -60, February 1967. 4

[6] B. J. Kang, K. R. Park, J. H. Yoo, and J. N. Kim. Multimodal biometric method that combines veins, prints, and shape of a finger. Optical Engineering, 50(1):017201-017201-13, January 2011. 1

[7] E. C. Lee, H. Jung, and D. Kim. New finger biometric method using near infrared imaging. Sensors, 11(3):2319-2333, 2011. 1

[8] E. C. Lee, H. C. Lee, and K. R. Park. Finger vein recognition using minutiabased alignment and local binary pattern-based feature extraction. International Journal of Imaging Systems and Technology, 19(3):179-186, 2009. 1

[9] S. Z. Li. Encyclopedia of Biometrics. Springer, New York, 1st edition, 2009. 4

[10] G. K. O. Michael, T. Connie, and A. B. J. Teoh. Touch-less palm print biometrics: Novel design and implementation. Image and Vision Computing, 26(12): $1551-1560,2008.1$

[11] T. Ojala, M. Pietikäinen, and D. Harwood. Performance evaluation of texture measures with classification based on Kullback discrimination of distributions. In Proceedings of the 12th IAPR International Conference on Pattern Recognition, volume 1, pages 582-585, October 1994. 1

[12] T. Ojala, M. Pietikäinen, and T. Mäenpää. Multiresolution gray-scale and rotation invariant texture classification with local binary patterns. IEEE Transactions on Pattern Analysis and Machine Intelligence, 24:971-987, 2002. 2

[13] M. 1. J. Swain and D. H. Ballard. Color indexing. International Journal of Computer Vision, 7:11-32, 1991. 4

[14] Y. Wang, K. Li, and J. Cui. Hand-dorsa vein recognition based on partition local binary pattern. In IEEE 10th International Conference on Signal Processing (ICSP), pages $1671-1674$, October 2010. 1

[15] Y. Wang, K. Li, J. Cui, L.-K. Shark, and M. Varley. Study of hand-dorsa vein recognition. In Advanced Intelligent Computing Theories and Applications, LNCS 6215, pages 490-498. Springer Berlin / Heidelberg, 2010. 1

[16] M. Watanabe, T. Endoh, M. Shiohara, and S. Sasaki. Palm vein authentication technology and its applications. In Proceedings of Biometrics Symposium, pages 37-38, 2005. 1

[17] B. Zhang, Y. Gao, S. Zhao, and J. Liu. Local derivative pattern versus local binary pattern: Face recognition with high-order local pattern descriptor. IEEE Transactions on Image Processing, 19(2):533 -544, 2010. 1, 2

[18] Y. Zhou and A. Kumar. Contactless palm vein identification using multiple representations. In 4th IEEE International Conference on Biometrics: Theory Applications and Systems (BTAS), pages 1- 6, September 2010. 1 